\title{
Mouse models of autism
}

\section{By Lev Osherovich, Senior Writer}

U.S. researchers have engineered mouse strains that model three relatively common genetic causes of autism spectrum disorder. ${ }^{1-3}$ The mice could help researchers figure out the similarities and differences between various forms of ASD and develop therapeutics tailored for specific subsets of ASD patients. Understanding the precise contribution of the three ASD-linked genetic alterations to the specific clinical manifestations of ASD will require the development of standardized behavioral and neurophysiological assays for the mutant mice.

The majority of ASD cases have no definitive cause. Only $20 \%-25 \%$ of cases are associated with genetic alterations, most of which are subtle mutations or changes in gene copy number of individual genes or small chromosomal regions. A handful of genetically-based ASD cases result from large-scale chromosomal abnormalities.

"Dozens of causes of autism have now been identified" through genomic methods, said Randall Carpenter. The challenge, he said, is to unravel how these genetic alterations lead to disease and to determine the relevance of these disease mechanisms for the majority of patients without genetic abnormalities.

Carpenter is cofounder, president and CEO of Seaside Therapeutics Inc., which is developing therapies for Fragile $\mathrm{X}$ syndrome and ASD.

Now, independent teams led by researchers at the University of California, Los Angeles, Harvard Medical School and Cold Spring Harbor Laboratory have found that mice with any of three distinct ASDlinked mutations or chromosomal abnormalities exhibit the classical behavioral signs of ASD, thus making it possible to undertake phenotypic screening for therapeutics.

The findings also reveal how ASD-related genes affect neurons and other brain cells involved in $\gamma$-aminobutyric acid (GABA) signaling and glutamate signaling.

\section{Locus pocus}

Two of the new mouse strains were designed to test the effects of deletions or duplications in a pair of chromosomal regions previously associated with ASD in human genomic studies.

A team led by Alea Mills, professor of genetics at Cold Spring Harbor, tackled the 16p11.2 chromosomal locus, which she said is deleted in "about $1 \%$ of autistic children, making it one of the strongest genetic factors" in ASD. The region contains 27 genes with a variety of functions, and it is unknown which contribute to ASD. ${ }^{1}$

The team made mice with one or three copies of the 16p11.2 locus, thus altering the levels of proteins encoded by the region.

She said her team "generated mice with altered dosage of the same 27 genes as patients with ASD, without any preconceived notions of what the molecular mechanisms might be."

Mice with only a single copy of the $16 \mathrm{p} 11.2$ locus had a high rate of neonatal mortality, and those that survived to adulthood had behavior that was reminiscent of ASD compared with wild-type littermates.

"In the heterozygous deletion, we saw sleep disorders, hyperactivity and highly repetitive behavior when we challenged these animals with a new environment," said Mills. "We also saw that eight regions of the brain are larger in the deletion mouse than in controls. This could be analogous to the increased head circumference measurements in ASD patients" compared with in individuals without ASD.

The team also made mice with an extra copy of the locus, but that strain behaved similarly to wild-type animals.

Results were published in the Proceedings of the National Academy of Sciences.

Mills now plans to drill down into the $16 \mathrm{p} 11.2$ region to find the specific genes responsible for the heterozygous knockout mouse's behavioral and neurophysiological phenotypes.

She has filed for a patent covering the $16 \mathrm{p} 11.2$ transgenic and heterozygous knockout mice.

\section{One gene at a time}

A Harvard Medical School team led by Matthew Anderson took a more targeted tack than Mills and focused on a single gene-ubiquitin protein ligase E3A (UBE3A;E6AP) - in a genomic region associated with another form of hereditary ASD. ${ }^{2}$

Anderson is assistant professor of neurology and pathology at Harvard Medical School and director of neuropathology at Beth Israel Deaconess Medical Center.

$U B E 3 A$ is part of the $15 q 11-13$ region, which occurs as a duplicate or triplicate in $1 \%-3 \%$ of patients with ASD.

Anderson hypothesized that this genetic alteration would be a good starting point for building ASD models. He said patients carrying extra copies of the 15q11-13 locus have a relatively straightforward form of ASD without the complex pathophysiological features of larger chromosomal alterations that cause the severe, syndromic forms of ASD.

"This is the most common genetic change in nonsyndromic autism patients," said Anderson.

Previous mouse studies by other researchers established that duplicating the entire region led to abnormal behavior. ${ }^{4}$ Anderson therefore hypothesized that increasing the number of copies of the Ube3a 
gene alone could result in an ASD-like condition in mice.

Compared with wild-type controls, mice with an extra copy of Ube $3 a$ had impaired social behavior, decreased vocalization in response to mouse social cues and increased repetitive self-grooming.

Anderson thinks these behavioral abnormalities are potentially indicative of ASD-like pathology and could thus be good assays for therapeutics. His next step is to compare the neurological characteristics of mice with extra copies of Ube $3 a$ against wild-type controls and Ube $3 a$ knockouts to understand what brain circuits are most affected by the gene.

Results were published in Science Translational Medicine. Anderson has filed patents on the discoveries, and the IP is available for licensing.

\section{Take a CNTNAP2}

In the third study, a UCLA team generated mice lacking the ASD-related gene contactin associated protein-like 2 (CNTNAP2). ${ }^{3}$

A rare loss-of-function mutation in that gene causes a syndrome of epilepsy, intellectual disability and autism. In 2008, genomewide association studies linked variations in CNTNAP2 to increased risk of ASD. . $^{5-7}$

Researchers led by Daniel Geschwind, professor of neurology, psychiatry and human genetics at UCLA, found that Cntnap2 knockout mice had behavioral problems including hyperactivity, repetitive behavior and awkward social interactions compared with wild-type controls. The knockout mice also were prone to seizures and had an abnormal electroencephalogram pattern.

Results were published in Cell and were not subject to patents.

\section{Model behavior}

One question going forward is whether studying the behavior of these three mouse models will be useful for understanding the disease process in the majority of ASD cases that lack such genetic alterations.

Robert Ring, VP of translation research at patient advocacy group Autism Speaks, cautioned that even though these studies present faithful models of the genetic lesions underlying some rare forms of ASD, the behavioral assays used by the researchers have yet to be validated.

"I'm not sure that we know how informative these mouse assays are," said Ring. "We can take highly penetrant genetic risk factors and make animal models, but how much these actually replicate the etiology of the neurodevelopmental problem in autism is unclear."

To resolve the question of how to interpret mouse behavior, Ring said Autism Speaks hopes to launch an effort to standardize phenotypic assays for mouse models.

"We have been thinking about funding a network of standardized assays to ensure there's consistency in the data," said Ring.

Geschwind, however, thinks the three new models could be relevant to a wide range of ASD cases. He said despite the distinct molecular causes, there was a surprising amount of similarity in the behavioral abnormalities of the three animal models. In addition, he said, those behavioral abnormalities resemble ASD in humans.

"Considering that there is no single mutation that causes more than $0.5 \%$ of cases of ASD, it's remarkable that in multiple models you can have genes which to some extent parallel what goes on in patients with ASD," said Geschwind.

Thus, the mice might point to common pathophysiological features among many ASD patients that could be counteracted with pharmacological intervention.

Ring said distinct neurological abnormalities can lead to symptoms that are similar from a behavioral standpoint but nonetheless may require different therapeutic interventions.

\section{Neuron the right track}

For example, Geschwind's team found that Cntnap2 knockouts had lower numbers of inhibitory GABAergic neurons than wild-type controls. This suggests agonizing the inhibitory GABAergic circuit could have beneficial effects.

Likewise, Anderson's team found evidence of decreased glutamatergic signaling in mice bearing extra copies of Ube3a.

"One of the exciting ideas in ASD is an imbalance of inhibitory and excitatory circuits," said Geschwind. It may be possible to correct this imbalance by treating with agonists or antagonists of whatever circuit is defective in any given mouse model, he noted.

As additional mouse models of ASD come online, Anderson said it may be possible to categorize the animals according to the nature of their signaling problems. Doing so could help identify subsets of ASD patients likely to respond to a given drug.

Indeed, Ring thinks the signaling alterations in the new mouse models might be better guideposts for therapeutic development than the behavioral phenotypes.

For now, drug developers will need more detailed information about the molecular defects caused by various ASD-associated chromosomal lesions before deciding how to interpret the mouse model data.

"We prioritize our research around single genes that we know are mutated and cause severe disorders," said Carpenter. "As you get into more complex situations like copy number variants, it gets harder to interpret the effects of these lesions on mouse behavior."

Seaside's most advanced candidate is arbaclofen (STX209), a GABA receptor agonist in Phase III testing for Fragile X syndrome. The company's STX107, a metabotropic glutamate receptor subtype 5 (mGluR5; GRM5) antagonist, is in Phase II testing for Fragile X syndrome.

Osherovich, L. SciBX 4(41); doi:10.1038/scibx.2011.1137

Published online Oct. 20, 2011

\section{REFERENCES}

1. Horev, G. et al. Proc. Natl. Acad. Sci. USA; published online Oct. 3, 2011; doi:10.1073/pnas.1114042108

Contact: Alea A. Mills, Cold Spring Harbor Laboratory, Cold Spring Harbor, N.Y.

e-mail: mills@cshl.edu

2. Smith, S.E.P et al. Science; published online Oct. 5, 2011; doi:10.1126/scitransImed.3002627

Contact: Matthew P. Anderson, Beth Israel Deaconess Medical Center, Boston, Mass.

e-mail: matthew_anderson@hms.harvard.edu

3. Peñagarikano, O. et al. Cell; published online Sept. 30, 2011; doi:10.1016/j.cell.2011.08.040 


\section{ANALYSIS}

Contact: Daniel H. Geschwind, University of California, Los Angeles, Calif. e-mail: dhg@ucla.edu

4. Nakatani, J. et al. Cell 137, 1235-1246 (2009)

5. Alarcón, M. et al. Am. J. Hum. Genet. 82, 150-159 (2008)

6. Arking, D.E. et al. Am. J. Hum. Genet. 82, 160-164 (2008)

7. Bakkaloglu, B. et al. Am. J. Hum. Genet. 82, 165-173 (2008)
COMPANIES AND INSTITUTIONS MENTIONED Autism Speaks, New York, N.Y.

Beth Israel Deaconess Medical Center, Boston, Mass. Cold Spring Harbor Laboratory, Cold Spring Harbor, N.Y. Harvard Medical School, Boston, Mass.

Seaside Therapeutics Inc., Cambridge, Mass. University of California, Los Angeles, Calif. 